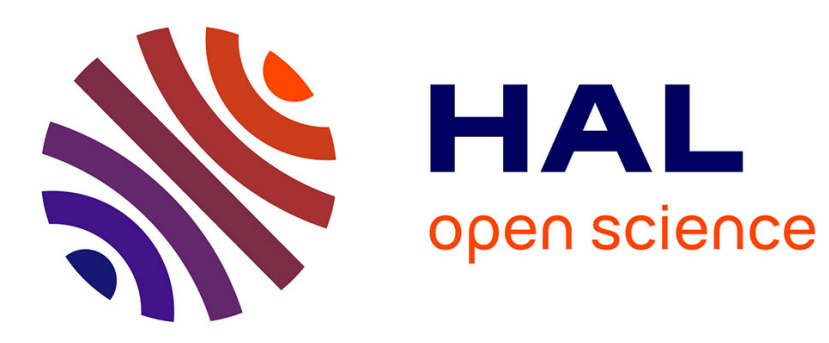

\title{
Characterization of bistable mechanisms for microrobotics and mesorobotics
}

\author{
Mouna Ben Salem, Hussein Hussein, Guillaume Aiche, Yassine Haddab, \\ Philippe Lutz, Lennart Rubbert, Pierre Renaud
}

\section{- To cite this version:}

Mouna Ben Salem, Hussein Hussein, Guillaume Aiche, Yassine Haddab, Philippe Lutz, et al.. Characterization of bistable mechanisms for microrobotics and mesorobotics: Comparison between microfabrication and additive manufacturing. Journal of Micro-Bio Robotics, 2019, 15 (1), pp.65-77. 10.1007/s12213-019-00113-3 . lirmm-02076914

\section{HAL Id: lirmm-02076914 https://hal-lirmm.ccsd.cnrs.fr/lirmm-02076914}

Submitted on 22 Mar 2019

HAL is a multi-disciplinary open access archive for the deposit and dissemination of scientific research documents, whether they are published or not. The documents may come from teaching and research institutions in France or abroad, or from public or private research centers.
L'archive ouverte pluridisciplinaire HAL, est destinée au dépôt et à la diffusion de documents scientifiques de niveau recherche, publiés ou non, émanant des établissements d'enseignement et de recherche français ou étrangers, des laboratoires publics ou privés. 


\title{
Characterization of bistable mechanisms for microrobotics and mesorobotics
}

\section{Comparison between microfabrication and additive manufacturing}

\author{
Mouna Ben Salem - Hussein Hussein • Guillaume Aiche • Yassine \\ Haddab • Philippe Lutz • Lennart Rubbert • Pierre Renaud
}

the date of receipt and acceptance should be inserted later

\begin{abstract}
The use of mechanical bistable structures in the design of microrobots and mesorobots has many advantages especially for flexible robotic structures. However, depending on the used fabrication technology, the adequacy of theoretical and experimental mechanical behaviors can vary widely. In this paper, we present the manufacturing results of bistable structures made with two extensively used contemporary technologies: MEMS and FDM additive manufacturing. Key issues of these fabrication technologies are discussed in the context of microrobotics and mesorobotics applications.
\end{abstract}

Keywords mechanical bistable structures · curved beams - MEMS · FDM additive manufacturing . microrobotics $\cdot$ mesorobotics

This research is partially supported by the Investissements d'Avenir (Labex CAMI ANR-11-LABX-0004). The authors would like to thank Gilles Bourbon and Patrice Le Moal from FEMTO-ST Institute for their contributions to the simulation, the development of MEMS process and for microfabrication.

M. Ben Salem, H. Hussein, G. Aiche and Y. Haddab

LIRMM, Université de Montpellier, CNRS Montpellier,

France

Tel: $+33 / 0467418585$

Fax: +33/0 467418500

E-mail: bensalem@lirmm.fr

$\mathrm{Ph}$. Lutz

FEMTO-ST Institute, Univ. Bourgogne Franche-Comté, CNRS, France

L. Rubbert, P. Renaud

ICube, INSA, Université de Strasbourg, CNRS Strasbourg, France

\section{Introduction}

One of the major paradigms in the design of very small robots is the use of flexible structures. At small scales, mechanisms are hard to design and assemble, and are generally advantageously replaced by such structures [1] Bistable structures are mechanical devices that have two stable states. They can be manufactured in conjunction with other parts of the microrobot in a monolithic manner. Using different micro-actuation principles, it is possible to build complete microrobots on a unique substrate using bistable modules [2]. Bistable structures offer a number of other advantages. They can be fabricated at low cost and built using various manufacturing processes. They are usable for energy or information storage. No energy is required to maintain a structure in one of its two stable states [2]. They are easily integrated into complex robotic architectures thanks to their planar shape and compactness [2]. They can be used in open-loop control without any sensor.

However, the use of bistable structures in the design of microrobots and mesorobots requires considering the modeling of their force-position characteristics and the limitations of the targeted manufacturing process. In the following, we consider more specifically two fabrication technologies: MEMS as it constitutes a technology that has been exploited for many microrobots developed over the last decade. A representative example of the use of bistable structures for the design of complete microrobots was presented in [2]. Each bistable structure is then used as an elementary module for the design of a high precision open loop controlled planar microrobots built using MEMS technology. The second technology is additive manufacturing. FDM (Fused Deposition Modeling) is of a particular interest for its wide availability, its potential low-cost 
and more importantly the possibility to produce parts with high performance materials such as PEEK, compatible for instance with the medical environment. This process indeed offers the possibility of manufacturing larger (mesoscale) structures if the application needs devices that can apply large forces or torques. Ion et al. propose meta structures based on bistable units [28]. In particular, microrobots for biomedical applications can be built thanks to the availability of biocompatible materials for FDM process. As an example of a medical application, a biomedical robot based on the bistable mechanism is developed in [29]. While MEMS are generally made of silicon, a material with very good elastic behavior which retains the mechanical properties of the material, FDM is being used for production of polymer parts, which has different properties after manufacturing.

In this paper we present elements on the design and operation of bistable structures for microrobotics and miniaturized robots. Section 2 presents the basics of modeling of curved beams, the main components of bistable structures. Section 3 is dedicated to the fabrication of curved beams using MEMS technology and section 4 presents a bistable mechanism manufactured by $3 \mathrm{D}$ printing and its experimental characterization. Manufacturing defects that arise from the miniaturization of parts which have dimensions close to the limits of the 3D printer are also being discussed as part of the manufacturing issues that comes with the use of this technology.

\section{Modeling of curved beams}

A curved beam structure is a component widely used in MEMS as a simple bistable mechanism that combines the advantages of passive holding and compliant mechanisms. Curved beams can be classified in three categories based on the fabrication process [3]:

- Precompressed curved beams, where the beam is fabricated rectilinear then it is compressed [3], [4], [5].

- Prestressed curved beams where during fabrication, the beam is buckled due to residual stresses which are added by heating or oxidation [6], [7].

- Preshaped curved beams where the beam is directly fabricated at the first buckling shape mode(see Fig. 1) without residual stresses [8], [9], [10].

The mechanical behavior of the precompressed curved beam is symmetric between two sides of buckling. However, the monolithic constraint and the difficulty to accurately set accurately the required buckling dimensions after fabrication make this solution difficult to use

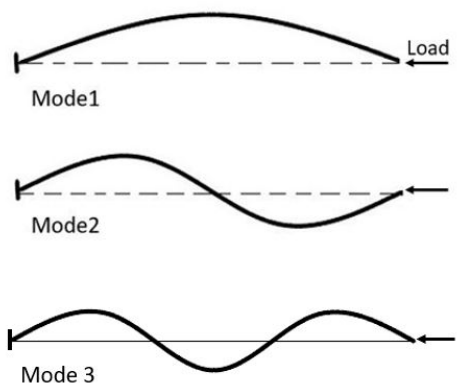

Fig. 1 Representation of the three first buckling modes

in MEMS. The prestressed curved beam makes the integration into a monolithic device easier, but the residual stress is difficult to control by fabrication [3]. For the pre-shaped curved beam, despite the fact that symmetry is lost and that bistability exists only under some conditions, fabrication and integration are simpler, specifically for MEMS applications. A solution used in the literature to improve the symmetry property of preshaped curved beams is to use hinged pre-shaped curved beam structure [11], [9], [12]. The thickness of a portion of the beam is then kept very low as compared to the overall beam thickness. This portion is usually designated as the elastic hinge. Local reinforcements are also proposed to be integrated with the pre-compressed length of a curved beam in [13] to adjust the postbuckling state and the snap-through properties. Few works can be found in literature that cover the modeling and design of a preshaped curved beam. Based on Lagrangian approach, Vangbo et al. [14] carried out one of the first studies on precompressed curved beams that takes compressibility into account for small deformations. Self-buckling behavior of microbeams in response to resistive heating was investigated by Chiao et al. [15]. Emam and Nayfeh examined in their studies [16], [17] the dynamics of precompressed curved beams. Cazottes [5] has investigated the bistability of a precompressed curved beam when actuated either by force or by moment. Elastic models for static and dynamic behaviors and experimental validation were presented by Camescasse in his thesis [4]. Chen et al. [18] showed the importance of extensible elastic theory in the modeling of a curved beam. As for preshaped curved beams, Qiu et al. in [9] presented analytical modeling for the snapping force behavior due to an applied lateral force on the middle length of the beam. The analytical results were confirmed by FEM simulations and experiments on microfabricated prototypes. Hussein et al. considered in [19] all the modes of buckling in the modeling 
of snapping forces and internal stresses. In comparison with FEM simulations and experiments on microfabricated prototypes, the consideration of high modes of buckling results in more accurate modeling mainly for the stress calculation. This calculation is important in order to consider the stress limits in the dimensioning of such a mechanism. Recently, the modeling expressions were further investigated in [30]. The effects of scaling the different dimensions and properties on the mechanical behavior of the curved beam were studied and a design methodology was presented. In most cases, the action to switch the curved beam is a lateral force applied at the middle of the beam. Otherwise, the action can be also a force applied in different points [4], an electromagnetic field [8], an electrostatic field [20], [21] or moments applied in determined locations on the beam [5], [22]. Park et al. [8] have presented analytical modeling of a preshaped curved beam when it is actuated by Laplace force distributed throughout the beam. The curved beam structure is being used in two mechanical forms: free or constrained, meaning that asymmetrical modes of buckling, such as mode 2 (presented in Fig. 1) can occur or not. The free structure consists generally of a single curved beam, while the constrained structure consists of two or more curved beams that are connected in the middle by a shuttle which is the moving part as shown in Fig. 2.

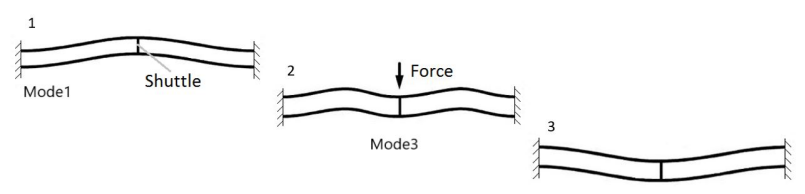

Fig. 2 Transition between the two stable positions (1 and 3 ) of two curved beams connected in the middle to a shuttle. Mode 3 appears during transition (2).

An example of constrained structure is the bistable device shown in Fig. 3 [12]. Two stop blocks are placed between the two initial stable sides of the shuttle in three different states (as-fabricated, at position 1 and at position 2). In the as-fabricated state, no residual stress or deformation energy is present, therefore, the shuttle is only stable without holding forces. The stop blocks are physical stops used to add holding forces at the two stable positions and to define accurately the distance between them. These positions do not change unless an external force is applied in the switching direction that is higher than the holding force. Otherwise, the stable positions can change due to any kind of disturbance, such as thermal expansion.

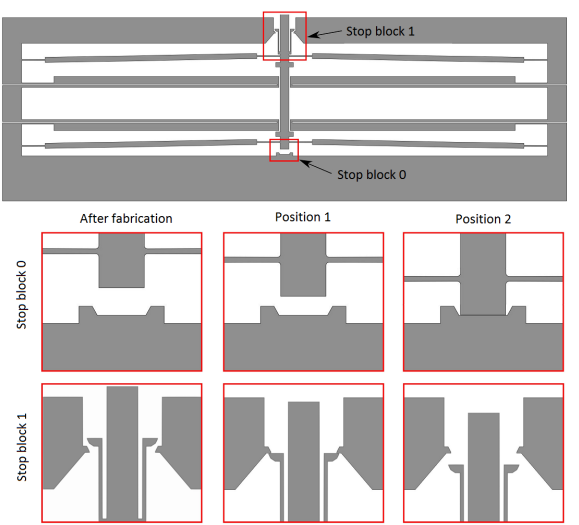

Fig. 3 Bistable structure with hinged curved beams and Ushaped actuators for switching [12].

A preshaped curved beam has the following characteristics, also presented in Fig. 4: thickness $t$, depth $b$, span $l$, deflection $d$, beam shape $w(x)$, initial height of buckling $h$, axial force $P$ and the applied lateral force at the middle $f$.

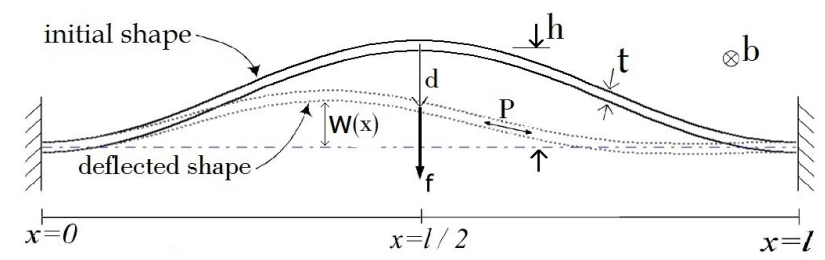

Fig. 4 Clamped-clamped curved bistable beam at the initial position and after deflection.

The governing equation of the buckling and postbuckling of a curved beam is developed by Timoshenko in his famous book [23].

$E I \frac{d^{4} w}{d x^{4}}+P \frac{d^{2} w}{d x^{2}}=0$

where $E$ is the Youngs modulus and $I$ is the quadratic moment of the cross section. The solution of (1) is an infinite sum of buckling shape modes $w_{j}$ where the constant modes $a_{j}$ are calculated in [9], [19] with a variational calculation after introducing the boundary conditions.

$w(x)=\sum_{j=1}^{\infty} a_{j} w_{j}(x)$

The axial force $P$ is constant along the beam. In the as-fabricated position, $P$ is equivalent to zero where no residual stress is present in the curved beam. Considering the case of two curved beams attached to a shuttle in the middle as in Fig. 2, the asymmetrical buckling 
shape mode cannot occur during deflection. Switching the curved beam to the other side of buckling, it becomes compressed and the axial force $P$ increases as long as the beam deflection $d$ gets closer to the middle axis. At a certain limit, $P$ reaches a defined value where bifurcation of solutions occurs in the variational calculation. At this point, the buckling shape mode 3 appears (see Fig. 1) in the shape of the curved beam and $P$ becomes constant. After a certain limit of deflection in the other side of buckling, mode 3 disappears and the axial force $P$ decreases. It is important to mention that $P$ does not reach the limit of bifurcation unless $Q=\frac{h}{t}$ is higher than 2.31. In terms of the snapping forces, behavior of the curved beam during deflection is nonlinear at the borders while it becomes linear when mode 3 appears in the middle zone of deflection. Figure 5 shows the snapping force curve with respect to deflection with highlights on remarkable points. As the mechanism is made by preshaped curved beams, it has an asymmetrical behavior.

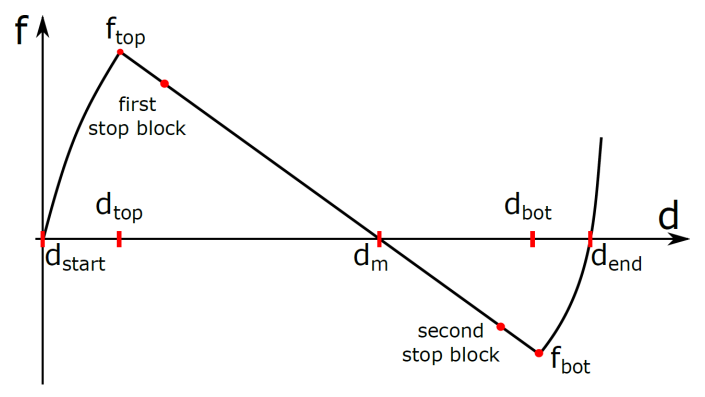

Fig. 5 Force-Displacement curve of a preshaped curved beam.

While the two stable positions correspond to minimal energy configuration, one is energy free (as fabricated position) and the second one is non-zero energy. In practice, stop blocks that define the holding forces at each stable position and the distance between them are placed in the linear domain in the central part of that characteristic. The expression of the snapping force evolution in that domain is given by:

$f=64 \pi^{2} \frac{E I h}{l^{3}}\left(\frac{4}{3}-\frac{d}{h}\right)$

The deflection zone where mode 3 appears is limited between $d_{t o p}$ and $d_{b o t}$.

$d_{\text {top }}, d_{\text {bot }}=h\left(\frac{28}{27} \pm \frac{2 \pi}{3} \sqrt{\frac{1}{6}+\frac{16}{81 \pi^{2}}-\frac{1}{Q^{2}}}\right)$

The calculation details of the snapping force in the nonlinear domain are presented in [19]. It is however important to mention that the snapping force curve can be approximated to a straight line for high values of $Q$.

\section{Microfabrication of curved beams}

The compliant nature of the curved beam offers many advantages such as the absence of friction and backlash, reduced wear, and low manufacturing costs. The use of silicon exhibits many additional advantages. The silicon has a nearly perfect elastic behavior with highly repeatable motion and without significant hysteresis or energy dissipation. It possesses in addition a long lifetime with reduced fatigue. This material is widely exploited in MEMS applications, its physical properties are well defined and the fabrication processes with silicon are well developed. Figure 6 illustrates an example of the main steps in a fabrication process. The fabrication is realized on SOI (Silicon On Insulator) wafers which is a common case for MEMS devices.
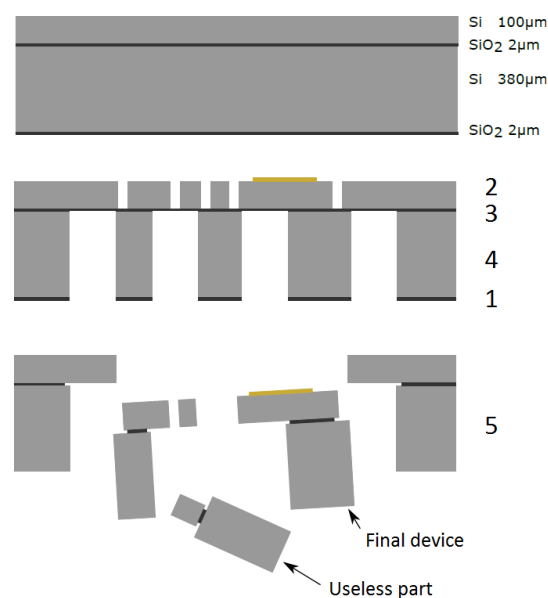

Fig. 6 Fabrication process steps. $\mathrm{SiO}_{2}$ hard mask etching on the backside (1), gold layer deposition (2), device and handle layers etched using DRIE process (3-4), structure releasing from the wafer using $\mathrm{HF}$ wet etching (5).

In the first step, the hard mask in the backside is lithographically patterned and etched using a photomask. In the second step, a gold layer is sputtered, then patterned using wet etching through a photoresist layer which is patterned with a second photomask. This layer serves as a conductive line for supplying the electrical components (electro thermal actuators are used in this device). In the third step, the device layer is patterned lithographically through a third photomask and then etched using a deep reactive ion etching (DRIE) process. In the fourth step, the handle layer is etched by DRIE through the backside $\mathrm{SiO}_{2}$ layer. Finally, the last step consists in releasing devices from the wafer using $\mathrm{HF}$ wet etching. Useless parts fall into the HF solution during etching. Figure 7 shows an example of obtained device. 


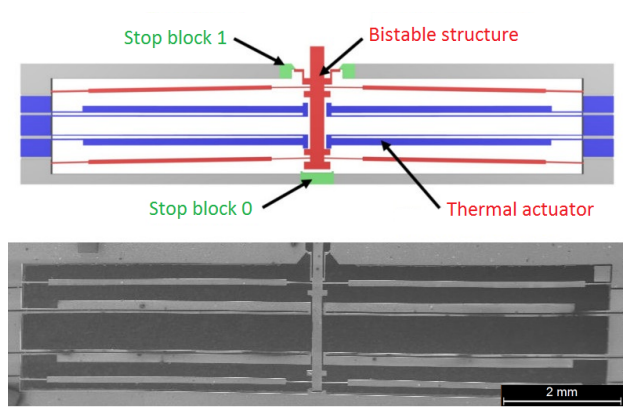

Fig. 7 A microfabricated bistable structure including thermal actuators and stop blocks.

Microfabrication details and characterization are given in [24]. The dimensions of the fabricated and characterised mechanism which is based on hinged preshaped curved beams (see Figure 8) are given in Table 1.

Table 1 Dimensions of the microfabricated bistable mechanism based on hinged preshaped curved beams

\begin{tabular}{|c||c|c|c|c|}
\hline Parameter & $l_{h b}$ & $t_{h b}$ & $b_{h b}$ & $h_{h b}$ \\
\hline Value & $500 \mathrm{~mm}$ & $15 \mathrm{~mm}$ & $100 \mu \mathrm{m}$ & $80 \mu \mathrm{m}$ \\
\hline
\end{tabular}

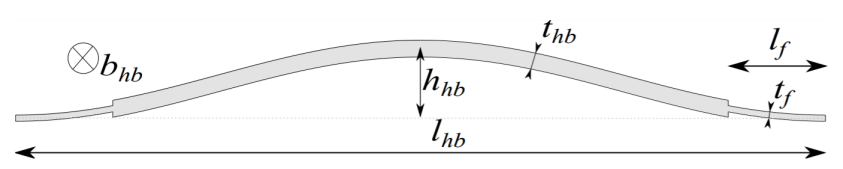

Fig. 8 A hinged preshaped curved beam at the initial position, with $t_{f}=20 \mu \mathrm{m}$ and $l_{f}=6.8 \mathrm{~mm}$

The behavior of the bistable mechanism shown in Fig. 7 and manufactured by microfabrication is characterized by experiments. Force-displacement characteristic is presented in Fig. 9. These measurements are made using an experimental setup adapted to the dimensions of the structures. The setup is composed of a force sensor (FemtoTools Microforce Sensing Probe) mounted on a robotic stage. When the latter is actuated, the force sensor triggers the bistable mechanism and returns the value of the force along with the associated displacement is recorded thanks to an interferometer (SIOS Metechnik GmbH) attached to the robotic stage. A dSPACE real time system is used to collect the data from the sensors (force and displacement) and sending control signals for the displacement of the robotic stage in real time. One can notice the good agreement between experimental and theoretical results [31].

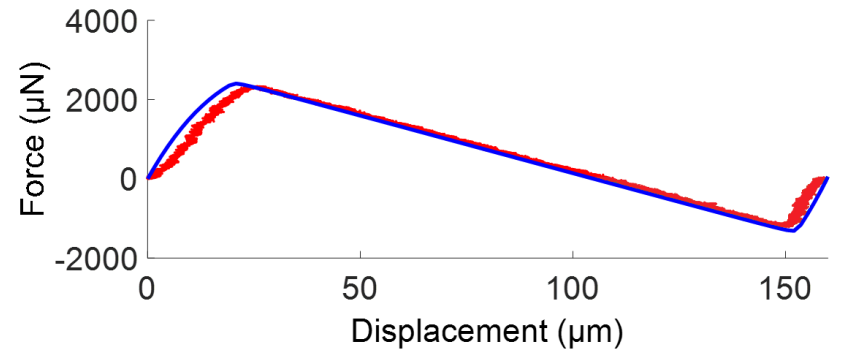

Fig. 9 Force-Displacement curves of a prototype composed of a hinged preshaped curved beams The blue curve is obtained by the analytical model and the red one is obtained by the experiments.

\section{$43 \mathrm{D}$ printing of curved beams}

\subsection{Towards 3D printed devices}

Since the last decade, the use of additively manufactured (AM) devices have become more and more important. As it goes adding material during the fabrication, it allows a new way of shaping the manufactured parts, unlike traditional machining. As it allows to prototype quickly, the trial and error method has become efficient, especially with polymer material, even though metal parts could also be manufactured using AM. However, as all manufacturing processes, the designer has to adapt his design to the additive manufacturing constraints. Focusing on fused deposition modeling (FDM) technology, a widespread AM technology, a filament material is deposited in plane layers. The final device consists of a stack of thin layers.

\subsection{Main process parameters}

A filament is made from extruded material, whose properties can be characterized. However, once heated and fused in layers, properties can be significantly affected. In additive manufacturing, geometry, material and process parameters are tightly linked. As the structure of the part depends on how the material is added, the manufactured part characteristics such as mechanical properties (aesthetic, roughness could also be mentioned) depends on the process parameters. For many years, researches have been conducted to understand and predict the behavior of the manufactured parts. Mohamed et al. [25] give a good overview of the different experiments presented in the literature, mainly for ABS material. Many parameters are analyzed, such as the layer thickness, the deposition orientation, the infill density and patterns, regarding their influence, mainly on mechanical properties. To understand how it can influence, the filament cross section is considered as a ho- 
mogeneous surface, whose mechanical properties could be known, as nominal ones. Considering the angle between the load and the cross section, it is possible to determine the mechanical properties. Since FDM allows changing many printing parameters, such as the previously mentioned, it affects the stiffness considering the load direction and the orientation of the built structure. Moreover, the fusion between the filaments on the same layer, and between layers is only partial, meaning that some void is remaining, reducing the stiffness and then mechanical properties.

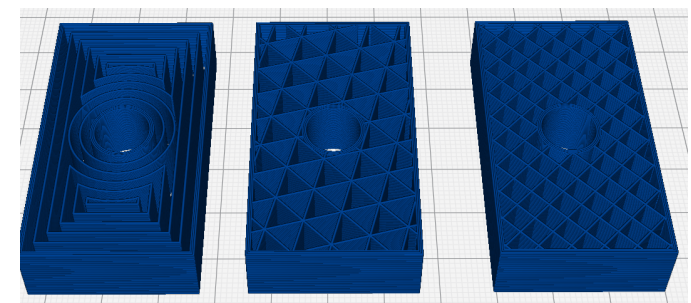

Fig. 10 Different infill patterns inducing different mechanical properties of the manufactured part: (left) concentric, (middle) triangle, (right) grid.

As a result, for instance, a linear pattern is stronger in the axis direction, but much weaker in the transverse direction, as delamination phenomena can appear, due to partial fusion between the deposited filaments. Changing pattern allows to improve part resistance in other directions (see Fig. 10). In short, the main engineering challenge is to find the best compromise between all the fabrication possibilities in order to best suit the part requirements. An example is shown in Fig. 11. Researches have been conducted to optimize performance. For instance, automatic orientation optimization is presented in [26].

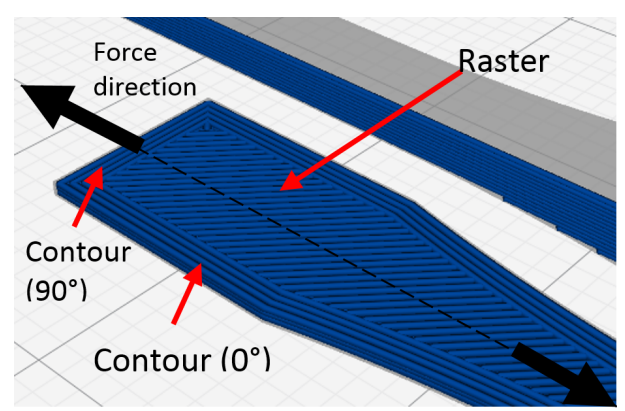

Fig. 11 Tensile characterization sample with different deposed filament orientation.

\section{$4.33 \mathrm{D}$ printed bistable mechanism}

From microscopic to mesoscopic, scale change could be a challenge especially while adapting the manufacturing technology to this change. When producing small structures with 3D printing, several limits can appear. As the stack of fused filament makes the resistance of a part, small parts using few layers can actually exhibit weaknesses leading to ruptures (see Fig. 12). For instance, it was noticed that some software favors the creation of close contours, instead of making open lines, (Fig. 12. B and C). Furthermore, discretization of the filament width is important if precision is needed in small dimensions. If width is not a multiple of the filament width (Fig. 12. D), lack or excess of material can be observed (Fig. 12. A).

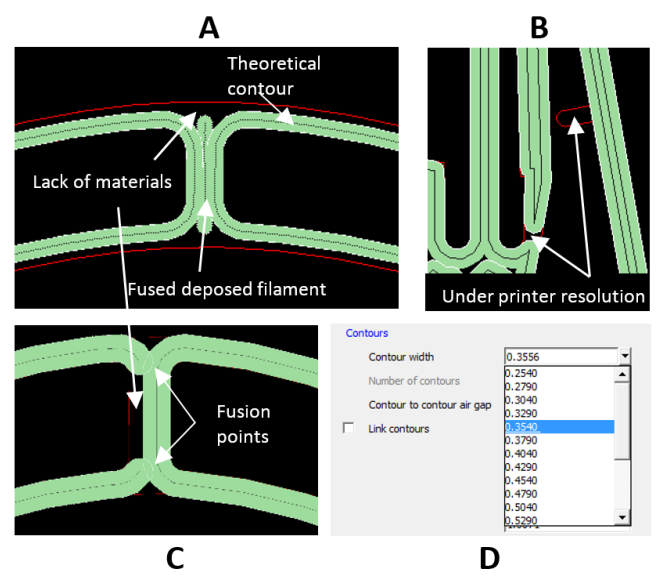

Fig. 12 Slicing and toolpath generation. Lack of material due to printer resolution: (A) incomplete filling, (B) lack of filling, (C) partial filament fusing inducing weakness, (D) available contour width.

In a first step, these difficulties have been avoided by the choice of dimensions that are far from the limits of the fabrication machine (Fortus $400 \mathrm{MC}$, Stratasys) and have proportions close to those introduced in section 2 which allows keeping the same ratio $Q$. The bistable mechanism manufactured by $3 \mathrm{D}$ printing and used in the experiments has the following dimensions presented in Table 2 and shown in Fig 13:

Table 2 Dimensions of the 3D printed bistable mechanism

\begin{tabular}{|c||c|c|c|c|}
\hline Parameter & $l$ & $t$ & $b$ & $h$ \\
\hline Value & $185 \mathrm{~mm}$ & $2 \mathrm{~mm}$ & $5 \mathrm{~mm}$ & $14 \mathrm{~mm}$ \\
\hline
\end{tabular}




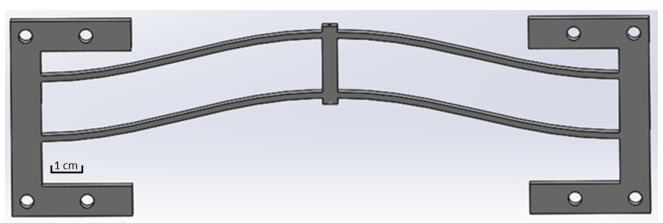

Fig. 13 CAD presentation of the bistable mechanism used for experimentation.

The bistable behavior can be presented by the model given in section 2 . The associated analytical model was also used to plot the force-displacement curve as shown in Fig. 16. In order to characterize the bistable mechanism, a dedicated setup has been built. It is made up of a laser-based displacement sensor (Keyence LK-H152) that has a measuring range of $\pm 40 \mathrm{~mm}$ with a resolution of $0.25 \mu \mathrm{m}$, a force sensor (Sauter FK50) that can apply a maximum force of $50 \mathrm{~N}$ with an accuracy of $0.02 N$ and a mounting bracket to fix the bistable mechanism and ensure the boundary conditions (see Fig. 14). An additional damping is added to prevent the movement of the mechanism during the experiment, since it was manufactured with an open frame. A force is applied using a linear displacement device to the double curved bistable beam and the corresponding displacement is measured using the laser sensor.

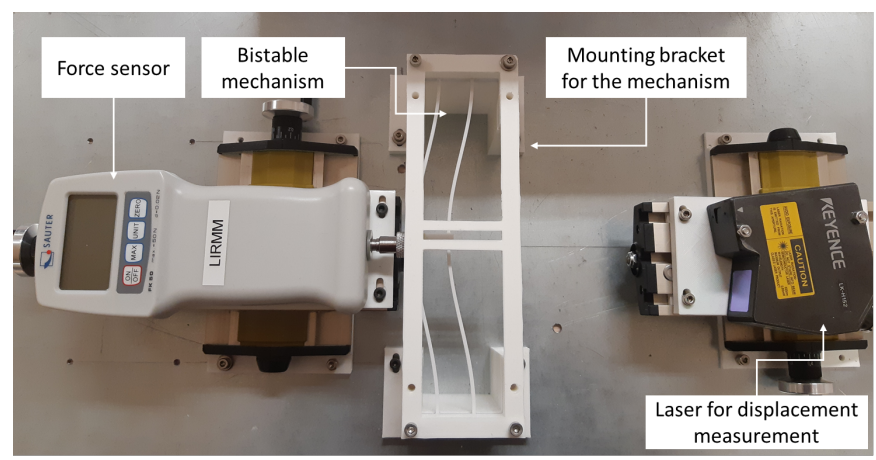

Fig. 14 Dedicated experimental setup, built for force displacement measurement.

During the experiments, the bistable mechanism passes as expected by the third mode of buckling, but when arriving at the equilibrium position the mechanism goes through an unexpected configuration (see Fig. 15. B). This shape is probably due to the fact that the stack of deposit filament does not create a fully solid part.
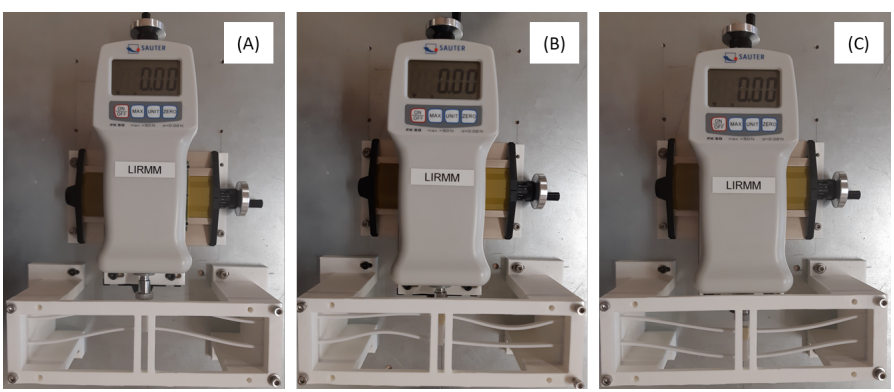

Fig. 15 Different positions of the bistable beam: (A) Stable position as fabricated, (B) Equilibrium position and (C) Second stable position.

The result of the experimental characterization is given in Fig. 16. The figure shows a comparison between the force-displacement curve obtained by the analytical model and the one obtained by the experiments. A remarkable difference in the amplitude of the force is noticed, however the displacements are in good agreement.

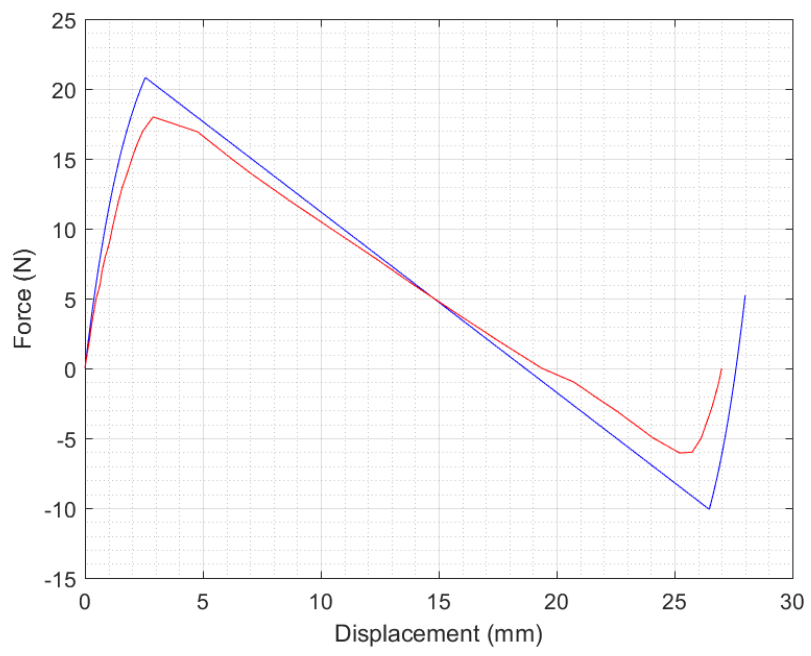

Fig. 16 Comparison of the force-displacement curves obtained by analytical model in blue and the experiments in red.

The dimensions of the manufactured structure are verified after 3D printing, therefore the Young's modulus (post-manufacturing) may be the origin of this difference considering that the expression of the force given in section 2 is directly proportional to the Young's modulus E. Therefore, the post-manufacturing Young's modulus is characterized in order to integrate it to the results of the analytical model.

To determine the influence of the additive manufacturing on the Young's modulus value, an experiment is performed on test beams manufactured in the same 
way as the bistable mechanism and using the same experimental setup (See Fig. 17).

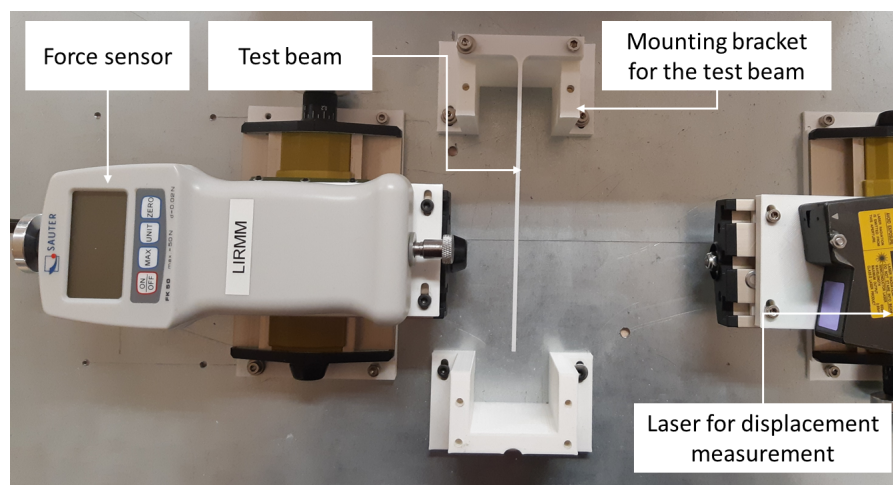

Fig. 17 Experimental setup used for the evaluation of the Young's modulus after manufacturing.

The measurements of forces and displacements made on the test beam allow the evaluation of the experimental Young's modulus using the following displacement expression of an end-loaded cantilever presented in Fig. 18.

$v=\frac{P L^{3}}{3 E I}$

where $E$ is the Young's modulus, $I$ is the quadratic moment of the cross section $P$ is the force applied at the tip of the cantilever, $L$ is the length of cantilever and $v$ is tip deflection.

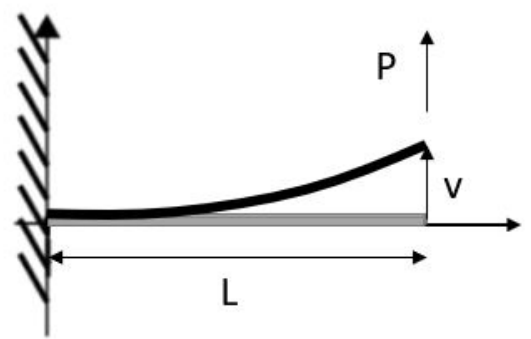

Fig. 18 Representation of an end-loaded cantilever.

The average value of the experimental Young's modulus is equal to $1772 \mathrm{MPa}$ compared to a theorical Young's modulus equal to $1944 \mathrm{MPa}$. By integrating this Young's modulus in the analytical model of the bistable mechanism, we obtain the results shown in Fig. 19. The error between the analytical and experimental amplitude decreases and the force-displacement curves show a better agreement between the presented theory in section 2 and the experiments.

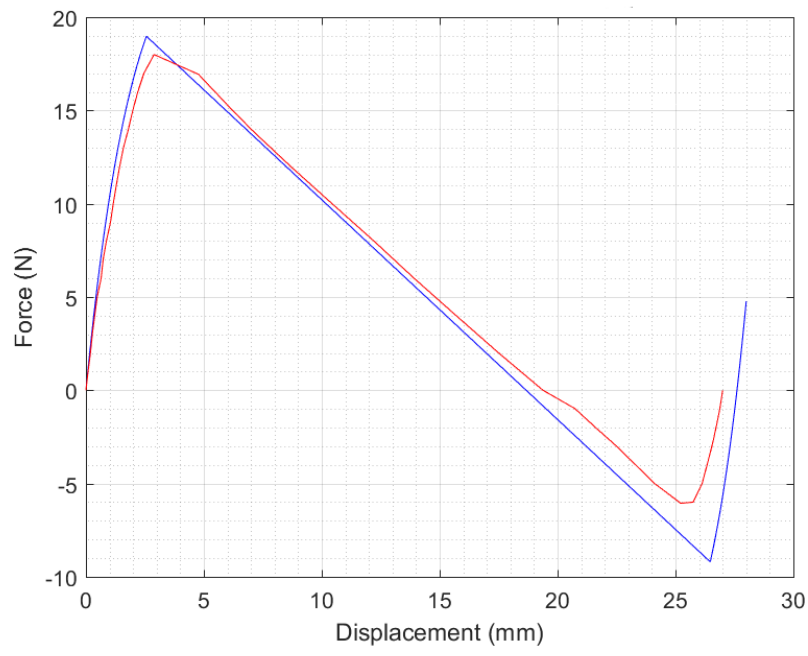

Fig. 19 Comparaison of the force-displacement curves obtained by analytical model with updated Young's modulus in blue and the experiments in red.

4.4 Miniaturization of a 3D printed bistable mechanism

In a second step, the fabricated structures are miniaturized. They have the dimensions given in Table 3 .

Table 3 Dimensions of the 3D printed miniaturized bistable mechanism

\begin{tabular}{|c||c|c|c|c|}
\hline Parameter & $l$ & $t$ & $b$ & $h$ \\
\hline Value & $18 \mathrm{~mm}$ & $0.35 \mathrm{~mm}$ & $2 \mathrm{~mm}$ & $2.4 \mathrm{~mm}$ \\
\hline
\end{tabular}

In this case, the manufacturing problems described previously and shown in Fig. 12 appear. The same 3D printer is used. The beam width consists of a single fused filament (Fig. 12. C), meaning that infill pattern cannot be changed. The second stable position of the bistable mechanism shows that deformation is different from theory (Fig. 20, right). Actually, some lack of material and plasticity induce unexpected behavior. Figure 12. $\mathrm{B}$ is taken from the tested structure in Fig. 20. It shows an example of a lack of material in the 3D printing process that we tried to improve as shown in the zoomed picture of the miniaturized bistable mechanism in Fig. 22.

The first experimental characterizations have shown that results are significantly different (Fig. 21). The figure shows a significant error, mainly about extremum force values and the evolution of the force-displacement curve obtained in the experiment. 

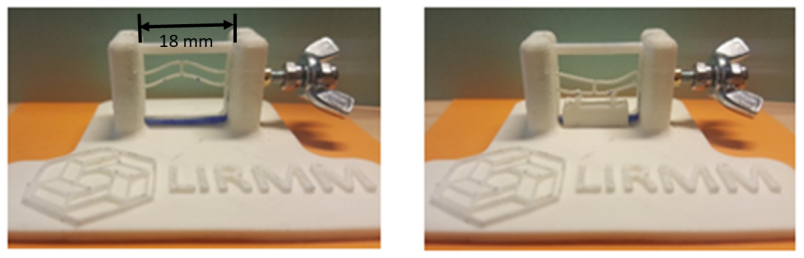

Fig. 20 Bistable curved beam (left) as shaped stable position, (right) other stable position limited by stop blocks.

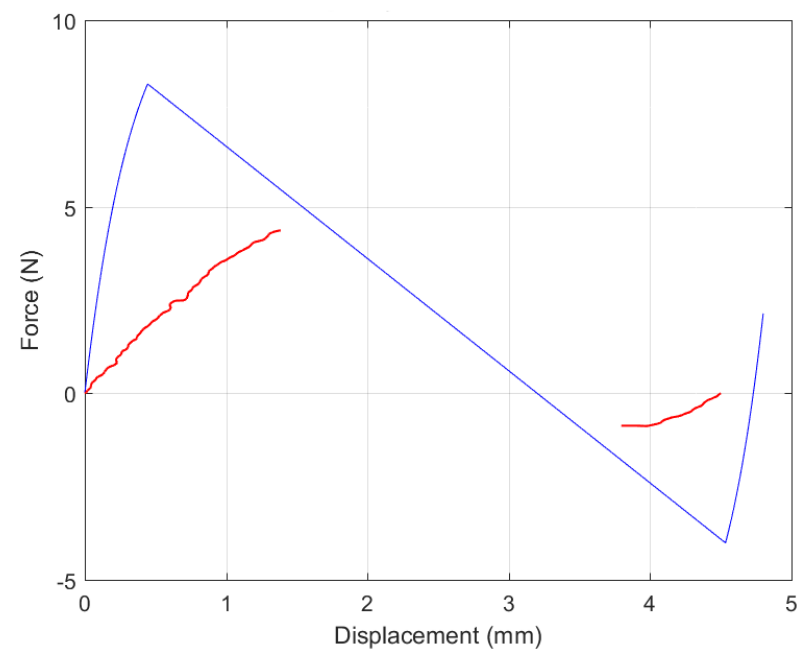

Fig. 21 Comparaison of the force-displacement curves obtained by analytical model in blue and the experiments in red of a miniaturized bistable mechanism

These results show that the greatest care must be taken when designing mesoscopic structures with FDM technology. The orientation and setting of the filament deposit can result in excess or lack of material (shown in Fig. 12) in the final part which can influence the behavior of the bistable mechanism (as shown in Fig. 15 and Fig. 20). Additionally, 3D printing changes the Young's modulus of the deposited filament which must be taken into consideration while characterizing the bistable mechanism.

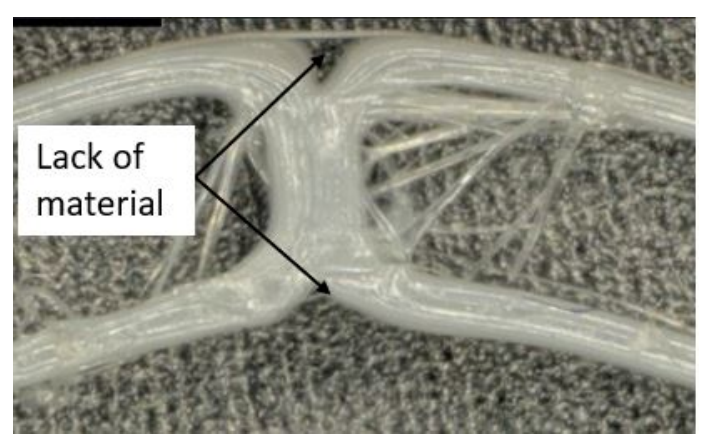

Fig. 22 A zoomed (x50) picture of the middle junction of the miniaturized bistable mechanism

\section{Discussion and conclusion}

In this paper, we presented the key concepts for the design and manufacture of bistable structures for microrobotic and mesorobotic applications. Two major manufacturing technologies were considered. The first one is microfabrication which allows the manufacturing of parts whith behaviors similar to modeling due to the elastic characteristics of the materials that are being used. However it remains an expensive fabrication process even for mass production. The second one is FDM 3D manufacturing which opens up a variety of possibilities for low cost devices that can be larger in size. However, the principle of additive manufacturing based on fused filament makes the mechanical behavior of the fabricated device difficult to predict and highly dependent on the trajectory control algorithms used in 3D printers. The results presented in section 4 show a difference in the force-displacement behavior of the 3D printed structure mainly due to the different Young's modulus after manufacturing which has been proven with characterization experiments. As for the miniaturized 3D printed bistable mechanism, other manufacturing defects appear which make the experimental behavior further away from the analytical result. These issues can be improved either by a better control of fabrication parameters. To summarize, different points of comparison between these two manufacturing methods are highlighted in the table. Future work will aim to define design rules for a better match between the theoretical and experimental models, considering the constraints of FDM, the printed specifications and its influence on the properties of the material. As another perspective for this work, a comparison study between FDM and other additive manufacturing process such as the Polyjet (developed by Stratasys). 


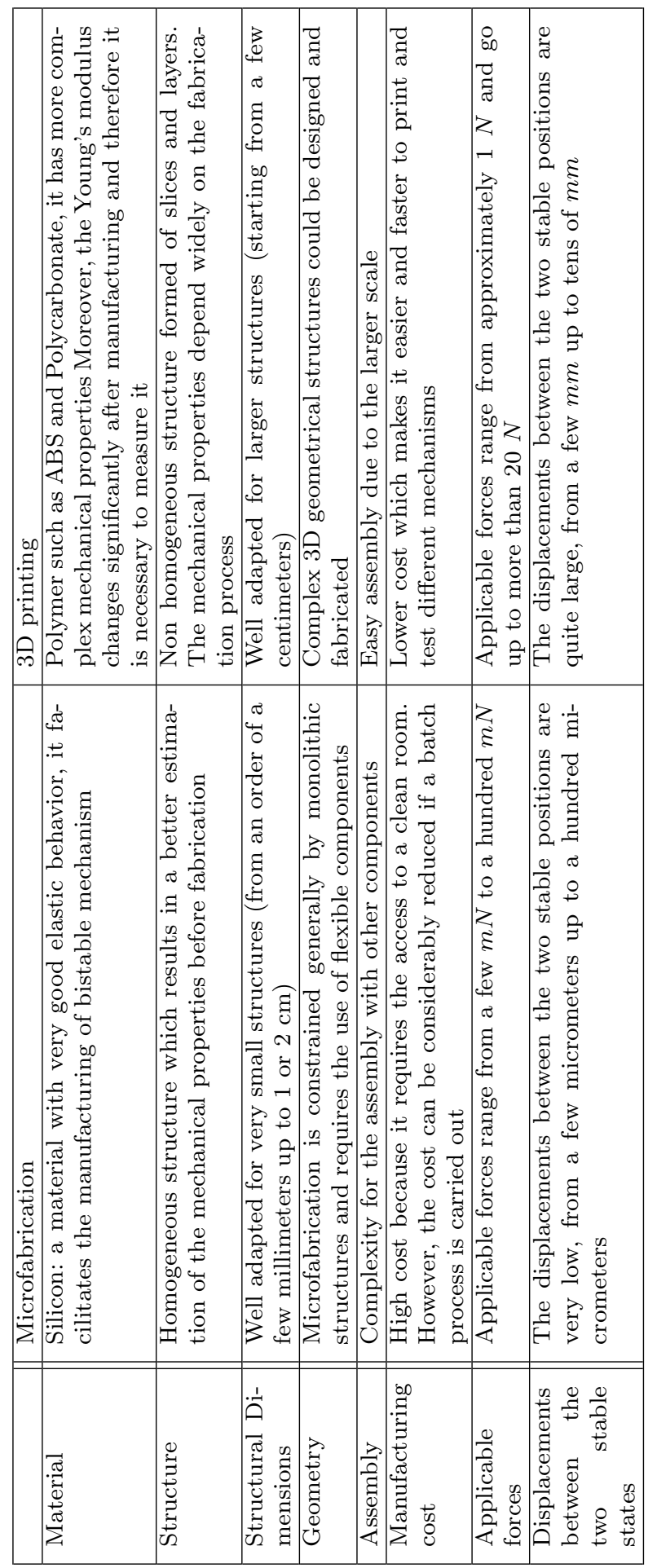




\section{References}

1. M. Grossard, N. Chaillet, and S. Rgnier, Flexible robotics: applications to multiscale manipulations, John Wiley \& Sons, (2013)

2. V. Chalvet, Y. Haddab and P. Lutz, A microfabricated planar digital microrobot for precise positioning based on bistable modules, IEEE - Transactions on Robotics, vol.29, Issue 3, pp. 641-649 (2013)

3. S. S. H. Zaidi, Z. Cherfi-Boulanger, and F. Lamarque, Contactless energy transfer and control strategy for bistable micro-actuator, Ph.D Thesis, University of Technology of Compigne, (2011)

4. B. Camescasse, Actionnements statique et dynamique dun mecanisme bistable : aspects modlisation, conception et exprimental, Ph.D. Thesis, Pierre and Marie University Curie, (2013)

5. P. Cazottes, Actionnement des systemes bistables: modélisation et études expérimentales, Ph.D Thesis, Pierre and Marie University Curie, (2009)

6. I. Z. Pane and T. Asano, Investigation on bistability and fabrication of bistable prestressed curved beam, Japanese Journal of Applied Physics, vol. 47, pp. 52-91, (2008)

7. B. Charlot, W. Sun, K. Yamashita, H. Fujita, and H. Toshiyoshi, In-plane bistable nanowire for memory devices, Design, Test, Integration and Packaging of MEMS/MOEMS, Symposium on. IEEE, pp. 254-258, (2008)

8. S. Park and D. Hah, Pre-shaped buckled-beam actuators: theory and experiments, Sensors and Actuators A: Physical, vol. 148, no. 1, pp. 186-192, (2008)

9. J. Qiu, J. Lang, and A. Slocum, A curved-beam bistable mechanism, vol. 13, no. 2, p. 137-146, (2004)

10. B.T. Liao, H.H. Shen, H.H. Liao, and Y.J. Yang, A bistable $2 \times 2$ optical switch monolithically integrated with variable optical attenuators, Optics express, vol. 17, no. 22, pp. 19919-19925, (2009)

11. B. D. Jensen, M. B. Parkinson, K. Kurabayashi, L. L. Howell, and M. S. Baker, Design optimization of a fully-compliant bistable micromechanism, ASME International Mechanical Engineering Congress and Exposition, vol. 48109, p. 21-25, (2001)

12. H. Hussein, V. Chalvet, P. Le Moal, G. Bourbon, Y. Haddab, and P. Lutz, Design optimization of bistable modules electrothermally actuated for digital microrobotics, IEEE/ASME International Conference on Advanced Intelligent Mechatronics AIM, pp. 1273-1278 (2014)

13. R. Gao, M. Li, Q. Wang, J. Zhao, and S. Liu, A novel design method of bistable structures with required snapthrough properties, Sensors and Actuators A: Physical, vol. 272, pp. 295-300 (2018)

14. M. Vangbo, An analytical analysis of a compressed bistable buckled beam, Sensors and Actuators A: Physical, vol. 69, no. 3, pp. 212-216, (1998)

15. M. Chiao and L. Lin, Self-buckling of micromachined beams under resistive heating, Journal of Microelectromechanical Systems, vol. 9, no 1, pp. 146-151 (2000)

16. S. A. Emam and A. H. Nayfeh, On the nonlinear dynamics of a buckled beam subjected to a primary-resonance excitation, Nonlinear Dynamics, vol. 35, no 1, pp. 1-17 (2004)

17. A. H. Nayfeh and S. A. Emam, Exact solution and stability of postbuckling configurations of beams, Nonlinear Dynamics, vol. 54, no 4, pp. 395-408 (2008)

18. J.S. Chen and H.W. Tsao, Static snapping load of a hinged extensible elastica, Applied Mathematical Modelling, vol. 37, no 18-19, pp. 8401-8408 (2013)
19. H. Hussein, P. Le Moal, G. Bourbon, Y. Haddab, and P. Lutz, Modeling and stress analysis of a pre-shaped curved beam: influence of high modes of buckling, International Journal of Applied Mechanics, vol. 7, no. 4, p. 1550055 , (2015)

20. S. Krylov and N. Dick, Dynamic stability of electrostatically actuated initially curved shallow micro beams, Continuum Mechanics and Thermodynamics, vol. 22, no. 6-8, pp. 445-468, (2010)

21. F. Tajaddodianfar, M. H. Yazdi, and H. N. Pishkenari, Dynamics of bistable initially curved shallow microbeams: Effects of the electrostatic fringing fields, IEEE/ASME International Conference on Advanced Intelligent Mechatronics AIM, pp. 1279-1283 (2014)

22. B. L. Stoimenov, J. M. Rossiter, and T. Mukai, Manufacturing of ionic polymer-metal composites (IPMCs) that can actuate into complex curves, Electroactive Polymer Actuators and Devices (EAPAD), International Society for Optics and Photonics, vol. 6524. (2007)

23. S. Timoshenko, Theory of elastic stability, McGraw-Hill, (1961)

24. Q. Chen, Y. Haddab, and P. Lutz, Microfabricated bistable module for digital microrobotics, Journal of MicroNano Mechatronics, vol. 6, pp. 1-12, (2010)

25. O. A. Mohamed, S. H. Masood, and J. L. Bhowmik, Optimization of fused deposition modeling process parameters: a review of current research and future prospects, Advances in Manufacturing, vol. 3, no 1, pp. 42-53 (2015)

26. E. Ulu, E. Korkmaz, K. Yay, O. B. Ozdoganlar, and L. B. Kara, Enhancing the Structural Performance of Additively Manufactured Objects Through Build Orientation Optimization, Journal of Mechanical Design, vol. 137, no 11, pp. 111410 (2015)

27. T. Letcher, B. Rankouhi, and Sina Javadpour, Experimental study of mechanical properties of additively manufactured ABS plastic as a function of layer parameters, International Mechanical Engineering Congress and Exposition, American Society of Mechanical Engineers, pp. V02AT02A018-V02AT02A018 (2015)

28. A. Ion, L. Wall, R. Kovacs, and P. Baudisch, Digital Mechanical Metamaterials, The 2017 CHI Conference on $\mathrm{Hu}-$ man Factors in Computing Systems, pp. 977-988 (2017)

29. M. Ben Salem, G.Aiche, L. Rubbert, P. Renaud and Y. Haddab, Design of a Microbiota Sampling Capsule using 3D-printed Bistable, 40th IEEE. International Engineering in Medicine and Biology Conference, pp. 4868-4871 (2018) 30. H. Hussein, P. Le Moal, R. Younis, G. Bourbon, Y. Haddab, and P. Lutz, On the design of a preshaped curved beam bistable mechanism, Mechanism and Machine Theory, vol. 131, pp. 204-217 (2019)

31. H. Hussein. Contribution to Digital Microrobotics: Modeling, Design and Fabrication of Curved Beams, U-shaped Actuators and Multistable Microrobots, PhD thesis, Bourgogne Franche-Comté University, (2015) 